\title{
2019 Reviewer Acknowledgement
}

\section{Editorial Office of Exploratory Research and Hypothesis in Medicine}

We thank the following reviewers for their contribution and support in 2019 .

\author{
Amal H Hamza \\ Saudi Arabia \\ Aliaë A R Mohamed-Hussein \\ Egypt \\ Alice M Richardson \\ Australia \\ Ahmad Bagheri Moghadam \\ Iran \\ Bohao Chen \\ United States \\ Szu-ta Chen \\ Taiwan \\ Chan Young Shin \\ South Korea \\ Cheng Guo \\ China \\ Hongzhi Xu \\ China \\ Chunjuan Song \\ United States \\ Wei Cui \\ China \\ Duan-Fang Liao \\ China \\ D Ortiz-Princz \\ Venezuela \\ Bo Li \\ China \\ Gang Sun \\ China \\ Jie Xu \\ China \\ Parag S. Bhalgat \\ India
}

Narinder Mehra
India

India

Germany

Nathan Martin Martin D'Cunha Australia

Xiaoling Yuan China

Ramya Kateel India

Rati Jani Australia

Shuwen Han China

Samie R. Jaffrey United States

Yunsheng Yang China

Yingmei Tang China

Anastasios Koulaouzidis United Kingdom

Feng-Wei Wang China

Won Fen Wong Malaysia

Hanjiang Wu China

Ce Yuan United States

Ji-Zong Zhao China

미: 10.14218/ERHM.2019.000RA 